\title{
AUTHOR INDEX Volume 26
}

Abdalla, A., see Eldakhly, N. M.

Aboul-Ela, M., see Eldakhly, N. M.

Akerkar, R., Plantié, M. and Trousset, F., Editorial

Alamaniotis, M., see Mali, A.

Antoniou, G. and Bikakis, A., Editorial

Antoniou, G., see Batsakis, S.

Ayed, R. B., see Zoghlami, M. A.

Bai, F., see Sun, Z.

Banbara, M., see Soh, T.

Batsakis, S., Tachmazidis, I. and Antoniou, G., Representing time and space for the semantic web

Bektas, J., Ibrikci, T. and Ozcan, I. T., Classification of real imbalanced cardiovascular data using feature selection and sampling methods: A case study with neural networks and logistic regression

Beynier, A., A multiagent planning approach for cooperative patrolling with non-stationary adversaries

Bikakis, A., see Antoniou, G.

Biswas, S. K., Chakraborty, M., Purkayastha, B., Roy, P. and Thounaojam, D. M., Rule extraction from training data using neural network

Bouchaffra, D., see Ykhlef, H.

Bourbakis, N., see Psarologou, A.

Bui, Q. T., see Pham, Q. D.

Cardiel, O., see Garrido, A. L.

Cerutti, F., Vallati, M. and Giacomin, M., An efficient Java-based solver for abstract argumentation frameworks: jArgSemSAT

Chaignaud, N., see Dubuisson Duplessis, G.

Chakraborty, M., see Biswas, S. K.

Chan, C.-K., Hao, J. and Leung, H.-F., Reciprocal social strategy in social repeated games and emergence of social norms

Chen, Y., see Qiu, Q.

Cordasco, G., see Esposito, A.

Costa, G. and Ortale, R., XML clustering by structure-constrained phrases: A fully-automatic approach using contextualized N-grams
26 (2017) 1750024

26 (2017) 1750024

26 (2017) 1702002

26 (2017) 1702004

26 (2017) 1702003

26 (2017) 1760015

26 (2017) 1750018

26 (2017) 1750017

26 (2017) 1760005

26 (2017) 1760015

26 (2017) 1750019

26 (2017) 1760018

26 (2017) 1702003

26 (2017) 1750006

26 (2017) 1760003

26 (2017) 1750012

26 (2017) 1750004

26 (2017) 1760022

26 (2017) 1750002

26 (2017) 1760009

26 (2017) 1750006

26 (2017) 1760007

26 (2017) 1760013

26 (2017) 1702001

26 (2017) 1760002 
da S. Arantes, J., da S. Arantes, M., Toledo, C. F. M., Júnior, O. T. and Williams, B. C., Heuristic and genetic algorithm approaches for UAV path planning under critical situation da S. Arantes, M., see da S. Arantes, J.

Dhiliphan Rajkumar, T., Raja, S. P. and Suruliandi, A., Users' 26 (2017) 1760008 26 (2017) 1760008 click and bookmark based personalization using modified agglomerative clustering for web search engine

26 (2017) 1730002

Dinakaran, S. and Ranjit Jeba Thangaiah, P., Ensemble method of effective adaboost algorithm for decision tree classifiers

Đonko, Dž., see Karahodža, B.

Dounias, G., see Tzanetos, A.

Dubuisson Duplessis, G., Pauchet, A., Chaignaud, N. and Kotowicz, J.-P., A conventional dialogue model based on dialogue patterns

26 (2017) 1760009

Eldakhly, N. M., Aboul-Ela, M. and Abdalla, A., Air pollution forecasting model based on chance theory and intelligent techniques

Esposito, A. M., see Esposito, A.

Esposito, A., Esposito, A. M., Troncone, A., Cordasco, G., Orlandini, A. and Tsoukalas, L., Editorial

Fakotakis, N., see Theodorou, T.

Fawcett, C., see Rizzini, M.

Fazakis, N., see Karlos, S.

Fiorino, H., see Ramoul, A.

Garrido, A. L., Sangiao, S. and Cardiel, O., Improving the generation of infoboxes from data Silos through machine learning and the use of semantic repositories

Gerevini, A. E., see Rizzini, M.

Giacomin, M., see Cerutti, F.

Guo, K., see Qiu, Q.

Guo, W., see Qiu, Q.

Haider, S., see Nasim, Z.

Hao, J., see Chan, C.-K.

He, Z., see Zhang, Y.

Hidri, M. S., see Zoghlami, M. A.

Hoos, H. H., see Rizzini, M.

Hu, Z.-P., see Sun, Z.

Ibrikci, T., see Bektas, J.

Júnior, O. T., see da S. Arantes, J.

Karahodža, B., Đonko, Dž. and Šupić, H., Modeling long-term user profile in collaborative filtering

26 (2017) 1750007

26 (2017) 1750021

26 (2017) 1750022

26 (2017) 1750024

26 (2017) 1702001

26 (2017) 1702001

26 (2017) 1750005

26 (2017) 1760006

26 (2017) 1750001

26 (2017) 1760021

26 (2017) 1760022

26 (2017) 1760006

26 (2017) 1750002

26 (2017) 1760013

26 (2017) 1760013

26 (2017) 1750023

26 (2017) 1760007

26 (2017) 1750016

26 (2017) 1750018

26 (2017) 1760006

26 (2017) 1750017

26 (2017) 1750019

26 (2017) 1760008

26 (2017) 1750021

Karlos, S., Fazakis, N., Kotsiantis, S. and Sgarbas, K.,

Self-trained stacking model for semi-supervised learning

26 (2017) 1750001

Kılıç, I. D. Y., Automated domain bias correction and its application in text-based personality analysis

26 (2017) 1760023

Kotowicz, J.-P., see Dubuisson Duplessis, G.

26 (2017) 1760009

Kotsiantis, S., see Karlos, S.

26 (2017) 1750001 
Křen, T., Pilát, M. and Neruda, R., Automatic creation of machine learning workflows with strongly typed genetic programming

26 (2017) 1760020

Ksouri, M., see Theljani, F.

Laabidi, K., see Theljani, F.

Lazaridis, A., see Theodorou, T.

Le, K. T., see Pham, Q. D.

Leung, H.-F., see Chan, C.-K.

Li, R., see Qiu, Q.

Liu, J., see Xue, X.

Mali, A. and Alamaniotis, M., Editorial

Mann, Z. Á. and Papp, P. A., Guiding SAT solving by formula partitioning

Marques-Silva, J., see Saber, T.

Melo, A., Völker, J. and Paulheim, H., Type prediction in noisy RDF knowledge bases using hierarchical multilabel classification with graph and latent features

Minaei-Bidgoli, B., see Seyyedi, S. H.

Mporas, I., see Theodorou, T.

Muthukumar, V., see Shahraki, F. F.

Nasim, Z. and Haider, S., ABSA toolkit: An open source tool for aspect based sentiment analysis

26 (2017) 1750010

26 (2017) 1750010

26 (2017) 1750005

26 (2017) 1750004

26 (2017) 1760007

26 (2017) 1760013

26 (2017) 1750013

26 (2017) 1702004

26 (2017) 1750011

26 (2017) 1760004

26 (2017) 1760011

26 (2017) 1750008

26 (2017) 1750005

26 (2017) 1750015

26 (2017) 1750023

26 (2017) 1760020

26 (2017) 1760017

Nguyen, M.-L., see Nguyen, M.-T.

Nguyen, M.-T., Tran, D.-V., Tran, C.-X. and Nguyen, M.-L., Exploiting user-generated content to enrich web document summarization

Nguyen, T. H., see Pham, Q. D.

Orlandini, A., see Esposito, A.

Ortale, R., see Costa, G.

Ouyang, Z. P., Wang, L. Z. and Wu, P. P., Spatial co-location pattern discovery from fuzzy objects

Ozcan, I. T., see Bektas, J.

Pan, S., see Kılıç, I. D. Y.

Papp, P. A., see Mann, Z. Á.

Pauchet, A., see Dubuisson Duplessis, G.

Paulheim, H., see Melo, A.

Pellier, D., see Ramoul, A.

Pereira, L. T. and Toledo, C. F. M., Speeding up search-based algorithms for level generation in physics-based puzzle games

Pesty, S., see Ramoul, A.

Pham, Q. D., Le, K. T., Nguyen, T. H., Pham, V. D. and Bui, Q. T., A constraint-based local search for offline and online general vehicle routing

26 (2017) 1760017

26 (2017) 1750004

26 (2017) 1702001

26 (2017) 1760002

26 (2017) 1750003

26 (2017) 1750019

26 (2017) 1760023

26 (2017) 1750011

26 (2017) 1760009

26 (2017) 1760011

26 (2017) 1760021

26 (2017) 1760019

26 (2017) 1760021

26 (2017) 1750004

26 (2017) 1750004

26 (2017) 1760020

Pilát, M., see Křen, T.

26 (2017) 1702002

Plantié, M., see Akerkar, R. 
Psarologou, A. and Bourbakis, N., Glossa - A formal language as a mapping mechanism of NL sentences into SPN state machine for actions/events association

26 (2017) 1750012

Purkayastha, B., see Biswas, S. K.

Qi, G., see Zhou, Z.

Qiu, Q., Guo, W., Chen, Y., Guo, K. and Li, R., Parallel multi-label propagation based on influence model and its application to overlapping community discovery

26 (2017) 1750006

26 (2017) 1760001

Rafiei, D., see Waqar, M.

Raja, S. P., see Dhiliphan Rajkumar, T.

Ramoul, A., Pellier, D., Fiorino, H. and Pesty, S., Grounding of HTN planning domain

Ranjit Jeba Thangaiah, P., see Dinakaran, S.

Regentova, E. E., see Shahraki, F. F.

Rizzini, M., Fawcett, C., Vallati, M., Gerevini, A. E. and Hoos, H. H., Static and dynamic portfolio methods for optimal planning: An empirical analysis

Roy, P., see Biswas, S. K.

Saber, T., Marques-Silva, J., Thorburn, J. and Ventresque, A., Exact and hybrid solutions for the multi-objective VM reassignment problem

Sadaoui, S., see Shil, S. K.

Sangiao, S., see Garrido, A. L.

Sekhavat, Y. A., Behavior trees for computer games

Seyyedi, S. H. and Minaei-Bidgoli, B., Enhancing effectiveness of dimension reduction in text classification

Sgarbas, K., see Karlos, S.

Shahraki, F. F., Yazdanpanah, A. P., Regentova, E. E. and Muthukumar, V., A trajectory based method of automatic counting of cyclist in traffic video data

26 (2017) 1760013

26 (2017) 1760010

26 (2017) 1730002

26 (2017) 1760021

26 (2017) 1750007

26 (2017) 1750015

26 (2017) 1760006

26 (2017) 1750006

26 (2017) 1760004

26 (2017) 1760016

26 (2017) 1760022

26 (2017) 1730001

26 (2017) 1750008

26 (2017) 1750001

26 (2017) 1750015

Shil, S. K. and Sadaoui, S., Multi-objective optimization in multi-attribute and multi-unit combinatorial reverse auctions

Slam, N., Slamu, W. and Wang, P., A prototype intelligent decision-support system with a unified planning and learning capabilities

Slamu, W., see Slam, N.

Soares, M., see Viana, P.

Soh, T., Banbara, M. and Tamura, N., Proposal and evaluation of hybrid encoding of CSP to SAT integrating order and log encodings

26 (2017) 1760016

26 (2017) 1750025

26 (2017) 1750025

26 (2017) 1760012

26 (2017) 1760005

Sotiropoulos, D. N. and Tsihrintzis, G. A., Artificial immune system-based classification in extremely imbalanced classification problems

26 (2017) 1750009

Spanjaard, O., see Weng, P.

26 (2017) 1760014

Stoilos, G., see Venetis, T.

26 (2017) 1760024

Sun, B., see Sun, Z.

26 (2017) 1750017 
Sun, Z., Hu, Z.-P., Wang, M., Bai, F. and Sun, B., Robust facial expression recognition with low-rank sparse error dictionary based probabilistic collaborative representation classification

Šupić, H., see Karahodža, B.

Suruliandi, A., see Dhiliphan Rajkumar, T.

Tachmazidis, I., see Batsakis, S.

Tamura, N., see Soh, T.

Theljani, F., Laabidi, K., Zidi, S. and Ksouri, M., An efficient density-based algorithm for data clustering

Theodorou, T., Mporas, I., Lazaridis, A. and Fakotakis, N., Data-driven audio feature space clustering for automatic sound recognition in radio broadcast news

Thorburn, J., see Saber, T.

Thounaojam, D. M., see Biswas, S. K.

Toledo, C. F. M., see da S. Arantes, J.

Toledo, C. F. M., see Pereira, L. T.

Tran, C.-X., see Nguyen, M.-T.

Tran, D.-V., see Nguyen, M.-T.

Troncone, A., see Esposito, A.

Trousset, F., see Akerkar, R.

Tsihrintzis, G. A., see Sotiropoulos, D. N.

Tsoukalas, L., see Esposito, A.

Tzanetos, A. and Dounias, G., Nature inspired optimization algorithms related to physical phenomena and laws of science: A survey

Utkin, L. V. and Zhuk, Y. A., Interval SVM-based classification algorithm using the uncertainty trick

Vallati, M., see Cerutti, F.

Vallati, M., see Rizzini, M.

Vassalos, V., see Venetis, T.

Venetis, T., Stoilos, G. and Vassalos, V., Rewriting minimizations for efficient query answering over ontologies

Ventresque, A., see Saber, T.

Viana, P. and Soares, M., A hybrid approach for personalized news recommendation in a mobility scenario using long-short user interest

Völker, J., see Melo, A.

Wang, L. Z., see Ouyang, Z. P.

Wang, M., see Sun, Z.

Wang, P., see Slam, N.

Waqar, M. and Rafiei, D., Characterizing users and tracking their activities in online classified ads

Weng, P. and Spanjaard, O., Functional reward Markov decision processes: Theory and applications

Williams, B. C., see da S. Arantes, J.

$\mathrm{Wu}$, P. P., see Ouyang, Z. P.

26 (2017) 1750017

26 (2017) 1750021

26 (2017) 1730002

26 (2017) 1760015

26 (2017) 1760005

26 (2017) 1750010

26 (2017) 1750005

26 (2017) 1760004

26 (2017) 1750006

26 (2017) 1760008

26 (2017) 1760019

26 (2017) 1760017

26 (2017) 1760017

26 (2017) 1702001

26 (2017) 1702002

26 (2017) 1750009

26 (2017) 1702001

26 (2017) 1750022

26 (2017) 1750014

26 (2017) 1750002

26 (2017) 1760006

26 (2017) 1760024

26 (2017) 1760024

26 (2017) 1760004

26 (2017) 1760012

26 (2017) 1760011

26 (2017) 1750003

26 (2017) 1750017

26 (2017) 1750025

26 (2017) 1760010

26 (2017) 1760014

26 (2017) 1760008

26 (2017) 1750003 
Xue, X. and Liu, J., A compact hybrid evolutionary algorithm for large scale instance matching in linked open data cloud

26 (2017) 1750013

Yazdanpanah, A. P., see Shahraki, F. F.

26 (2017) 1750015

Ykhlef, H. and Bouchaffra, D., Induced subgraph game for ensemble selection

26 (2017) 1760003

Zhang, X. and Zhang, X., Cross entropy method meets local search for continuous optimization problems

26 (2017) 1750020

Zhang, X., see Zhang, X.

Zhang, Y. and He, Z., Video object segmentation through deep

26 (2017) 1750020 convolutional networks

26 (2017) 1750016

Zhou, Z. and Qi, G., GEL: A platform-independent reasoner

for parallel classification with OWL EL ontologies using

graph representation

26 (2017) 1760001

Zhuk, Y. A., see Utkin, L. V.

26 (2017) 1750014

Zidi, S., see Theljani, F.

26 (2017) 1750010

Zoghlami, M. A., Hidri, M. S. and Ayed, R. B., Consensus-driven cluster analysis: Top-down and bottom-up based split-and-merge classifiers

26 (2017) 1750018 